Document downloaded from:

http://hdl.handle.net/10251/146166

This paper must be cited as:

Helal-Neto, E.; Sánchez-Cabezas, S.; Sancenón Galarza, F.; Martínez-Máñez, R.; SantosOliveira, R. (10-0). Indirect calculation of monoclonal antibodies in nanoparticles using the radiolabeling process with technetium 99 metastable as primary factor: Alternative methodology for the entrapment efficiency. Journal of Pharmaceutical and Biomedical Analysis. 153:90-94. https://doi.org/10.1016/j.jpba.2018.02.017

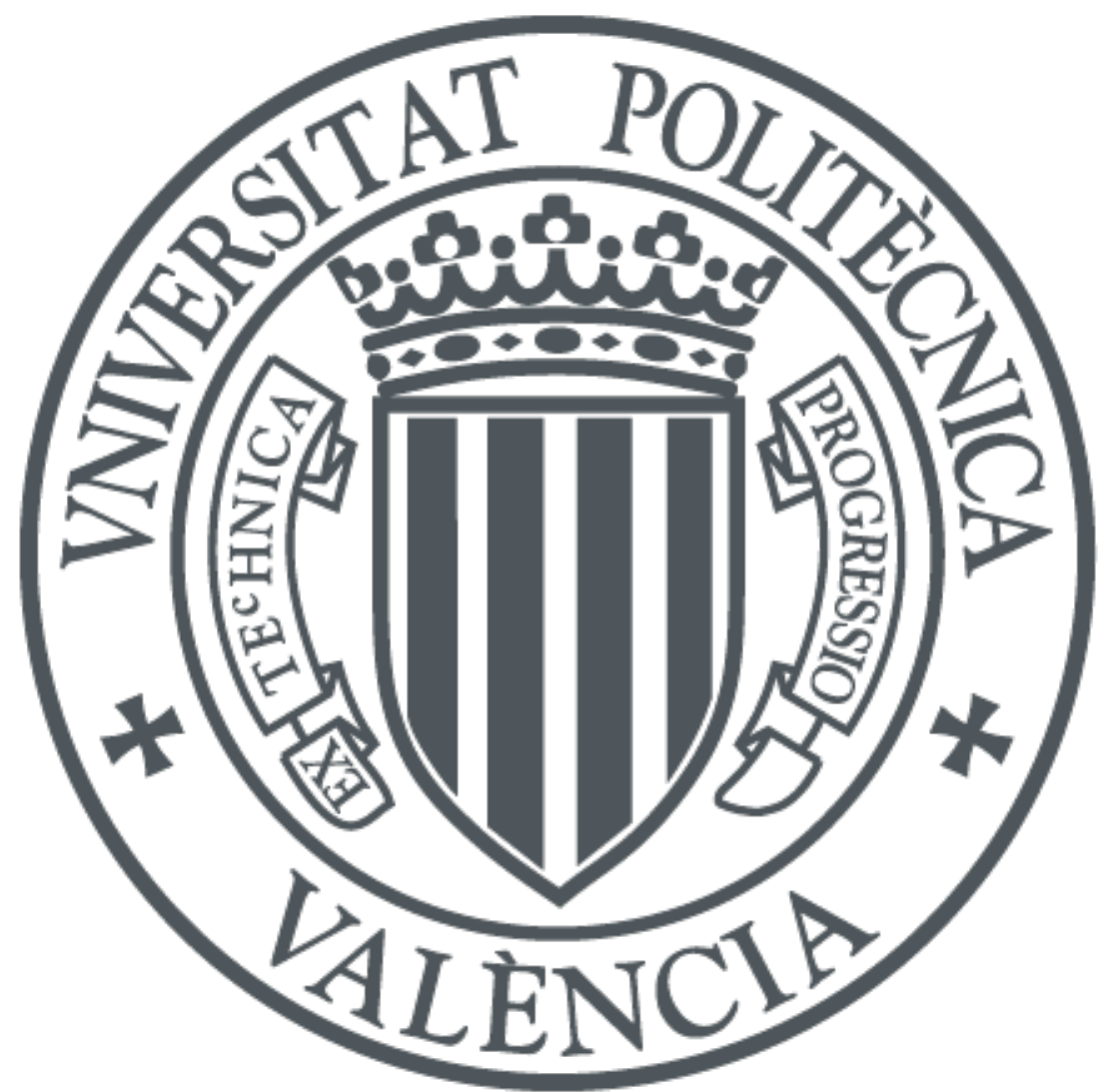

The final publication is available at

https://doi.org/10.1016/j.jpba.2018.02.017

Copyright Elsevier

Additional Information 


\section{Indirect calculation of monoclonal antibodies in nanoparticles using the radiolabeling process with Technetium 99 metastable as primary factor: alternative methodology for the entrapment efficiency}

Edward Helal-Neto ${ }^{1}$, Santiago Sánchez Cabezas ${ }^{2,3,4}$, Félix Sancenón²,3,4, Ramón Martínez-Máñez ${ }^{2,3,4}$, Ralph Santos-Oliveira ${ }^{1,5}$

1- Brazilian Nuclear Energy Commission, Nuclear Engineering Institute, Rio de Janeiro, Brazil

2- Instituto Interuniversitario de Investigación de Reconocimiento Molecular y Desarrollo Tecnológico (IDM), Universitat Politècnica de València, Universitat de València. Camino de Vera s/n, 46022, Valencia, Spain

3- Departamento de Química, Universidad Politécnica de Valencia, Camino de Vera s/n, 46022, Valencia, Spain.

4- CIBER de Bioingeniería, Biomateriales y Nanomedicina (CIBER-BBN).

5- Zona Oeste State University, Laboratory of Nanoradiopharmaceuticals, Rio de Janeiro, Brazil

All correspondence to

Dr Ralph Santos-Oliveira

Brazilian Nuclear Energy Commission

Nuclear Engineering Institute

Rio de Janeiro-Brazil

roliveira@ien.gov.br 


\section{Graphical Abstract}

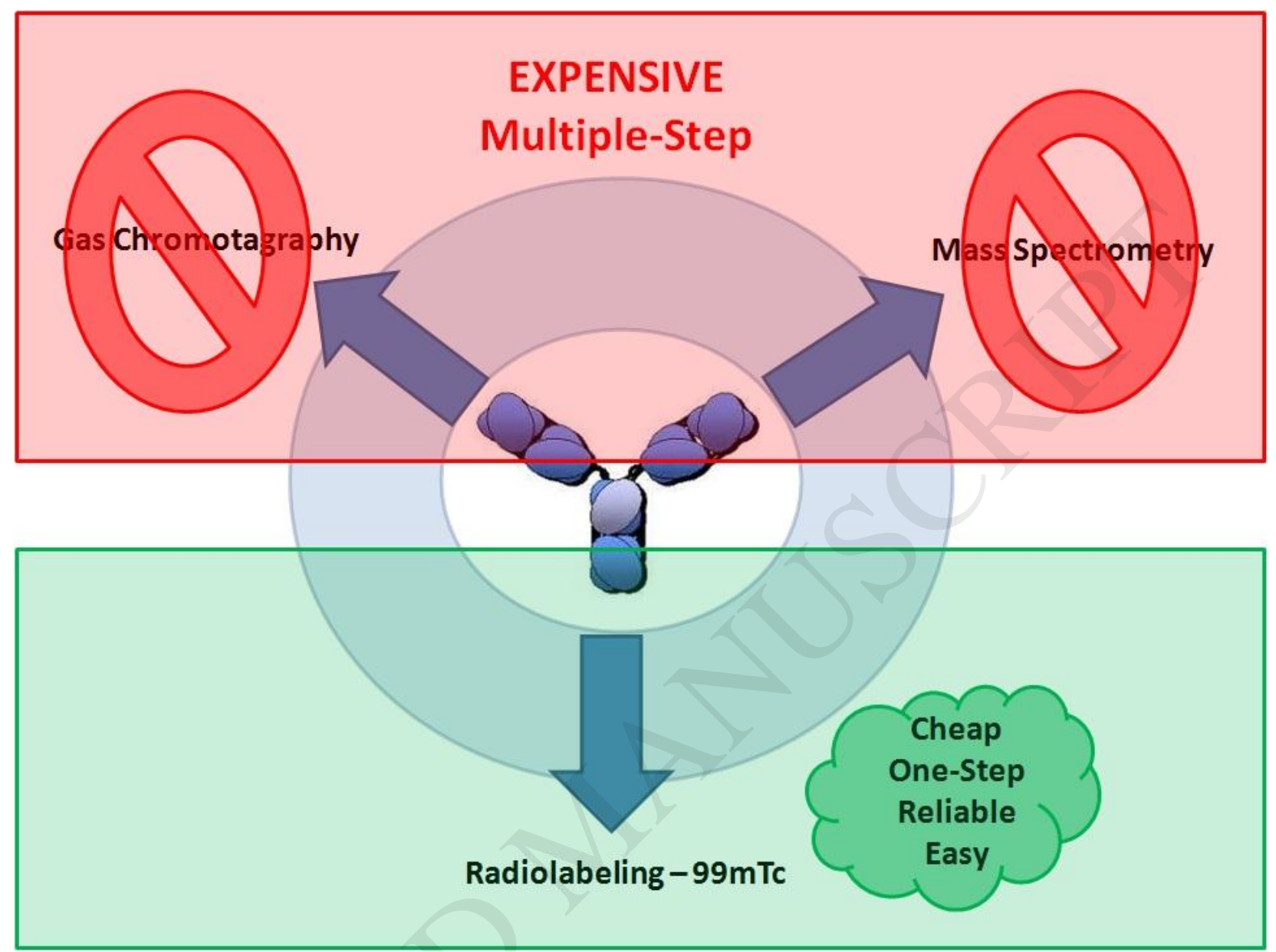

HIGHLIGHTS

- Novel methodology for the development of magnetic core mesoporous silica

- Radiolabeling process of monoclonal antibodies with $99 \mathrm{mTc}$

- Efficient methodology to calculate the Incorporation of monoclonal antibodies into nanoparticles by radiolabeling process 


\section{Abstract}

The use of monoclonal antibodies (Mab) in the current medicine is increasing. Antibody-drug conjugates (ADCs) represents an increasingly and important modality for treating several types of cancer. In this area, the use of Mab associated with nanoparticles is a valuable strategy. However, the methodology used to calculate the Mab entrapment, efficiency and content is extremely expensive. In this study we developed and tested a novel very simple one-step methodology to calculate monoclonal antibody entrapment in mesoporous silica (with magnetic core) nanoparticles using the radiolabeling process as primary methodology. The magnetic core mesoporous silica were successfully developed and characterized. The PXRD analysis at high angles confirmed the presence of magnetic cores in the structures and transmission electron microscopy allowed to determine structures size (58.9 \pm $8.1 \mathrm{~nm})$. From the isotherm curve, a specific surface area of $872 \mathrm{~m}^{2} / \mathrm{g}$ was estimated along with a pore volume of $0.85 \mathrm{~cm}^{3} / \mathrm{g}$ and an average pore diameter of $3.15 \mathrm{~nm}$. The radiolabeling process to proceed the indirect determination were well-done. Trastuzumab were successfully labelled (>97\%) with Tc-99m generating a clear suspension. Besides, almost all the Tc-99m used (labelling the trastuzumab) remained trapped in the surface of the mesoporous silica for a period as long as 8 hours. The indirect methodology demonstrated a high entrapment in magnetic core mesoporous silica surface of Tc-99m-traztuzumab. The results confirmed the potential use from the indirect entrapment efficiency methodology using the radiolabeling process, as a one-step, easy and cheap methodology. 
Keywords: nanoparticles, Magnetic Core Mesoporous Silica Nanoparticles doped with Trastuzumab and labeled with Tc-99m, cancer, oncology, smart device.

\section{Introduction}

The use of monoclonal antibodies (Mabs) in the current medicine is increasing each day. Monoclonal antibody-based treatment is used in a great variety of disease, with especial attention in oncology [1-3]

Antibody-drug conjugates (ADCs) are monoclonal antibodies attached to biologically active drugs by chemical linkers with labile bonds. It represents an increasingly and important modality for treating several types of cancer. The impact of ADCs in this field is due the exquisite specificity of antibodies that deliver the conjugated cytotoxic agent to targeted tumor cells, thus reducing the systemic toxicity associated with traditional chemotherapeutic treatments. ADCs are distinguished on the basis of the drug, linker, and also the amino acid residue of attachment on the antibody [4-6]. In the field of ADC, the use of Mab associated with nanoparticles is very common [7-9]. In this specific ADC case the calculation of Mab entrapment efficiency is of vital importance and in many cases requires a specific and expensive methodology.

The primary method to evaluate the mAb purification can be made by Protein A chromatography, followed by two or three subsequent chromatographic polishing steps [10]. In this case the chromatographic column continues to be favored, and has been the focus of continuous improvements 
through development of higher capacity resins and use of mixed-mode sorbents. Another methodology involves mass spectroscopy or mass spectroscopy-associated liquid chromatography [11,12]. In both cases, the methodology used to calculate the Mab entrapment, efficiency and content is extremely expensive. In this study we developed and tested a novel and very simple one-step methodology to calculate monoclonal antibody entrapment efficiency in mesoporous silica nanoparticles using the radiolabeling process as primary methodology.

\section{Materials and methods}

2.1. Preparation of magnetic core mesoporous silica nanoparticles (MSN)

\subsubsection{Reagents and materials}

Iron(III)chloride hexahydrate, iron(II)tetrachloride hexahydrate, oleic acid, hexadecyltrimethylammonium bromide (CTAB) and tetraethyl orthosilicate (TEOS) were purchased form Sigma. Ammonia solution (32\%), ethanol and ethyl acetate were purchased from Scharlau. Chloroform was obtained from Acros Organics. Distilled water was used in all reactions.

\subsubsection{Synthesis of oleate-coated iron oxide nanoparticles}

Iron oxide nanoparticles $\left(\mathrm{Fe}_{3} \mathrm{O}_{4}\right.$ magnetite nanocrystals) were obtained by a modified coprecipitation method [1]. Briefly, $12 \mathrm{~g}$ of iron(III)chloride hexahydrate were mixed with $4.9 \mathrm{~g}$ of iron(II)chloride tetrahydrate in $50 \mathrm{ml}$ of water at $80{ }^{\circ} \mathrm{C}$ 
under a flow of argon and mechanical stirring. Ammonia solution 32\% (19.53 $\mathrm{ml}$ ) was carefully added and the mixture turned completely dark. Oleic acid $(2.13 \mathrm{ml})$ was added after $30 \mathrm{~min}$ and the reaction was left stirring at $80{ }^{\circ} \mathrm{C}$ for another $90 \mathrm{~min}$. The reaction was cooled down and centrifuged at $9500 \mathrm{rpm}$ for 10 min. The resulting black precipitate was washed three times with distilled water and three times with ethanol and then dried under vacuum overnight. In order to prevent their oxidation, the oleate-coated iron oxide nanoparticles were kept in chloroform giving a dark brown ferrofluid.

\subsubsection{Synthesis of magnetic core MSNs}

In a typical procedure, $100 \mathrm{mg}$ of CTAB were dissolved in $10 \mathrm{ml}$ of water, followed by addition of $0.74 \mathrm{ml}$ of the ferrofluid $(8.88 \mathrm{mg} / \mathrm{ml})$. The mixture was placed in a probe sonicator (Branson 450 Sonifier) for 2 min, giving an oil-inwater emulsion. Then, the mixture was heated to $65{ }^{\circ} \mathrm{C}$ to evaporate the chloroform and achieve an effective phase transfer from chloroform to water. The resulting transparent aqueous suspension was added to a solution of $30 \mathrm{ml}$ of water and $0.548 \mathrm{ml}$ of ammonia (32\%), which was then, heated up to $75{ }^{\circ} \mathrm{C}$. Then, $0.5 \mathrm{ml}$ of tetraethyl orthosilicate (TEOS) was added dropwise followed by addition of $3 \mathrm{ml}$ of ethyl acetate. The reaction was stirred at $350 \mathrm{rpm}$ and $75 \stackrel{\circ}{ } \mathrm{C}$ during $3 \mathrm{~h}$. Then, the reaction mixture was placed on an ice bath and the nanoparticles were collected by centrifugation (9500 rpm, $10 \mathrm{~min}$ ). Afterward, the sample was washed with ethanol twice and dried under vacuum overnight. The final magnetic core MSNs were calcined in air at $550{ }^{\circ} \mathrm{C}$ for $5 \mathrm{~h}$.

\subsection{Characterization of magnetic core MSNs}




\subsubsection{Powder X-ray diffraction}

The synthesised materials were characterised by powder X-ray diffraction (PXRD), transmission electron microscopy (TEM) and $\mathrm{N}_{2}$ adsorption-desorption analysis. PXRD measurements were obtained using a Bruker AXS D8 Advance diffractometer equipped with CuKa radiation and working at $40 \mathrm{kV} / 40 \mathrm{~mA}$. PXRD measurements were performed at high angle $\left(2 \theta=15^{\circ}-68^{\circ}\right)$ and low angle range $\left(2 \theta=1.3^{\circ}-8 \cdot 3^{\circ}\right)$.

\subsubsection{Transmission electron microscopy}

TEM images were taken on a $100 \mathrm{kV}$ JEOL JEM-1010 microscope operated with AMT image capture engine software. TEM samples were prepared by adding $10 \mu \mathrm{l}$ of nanoparticles suspended in distilled water onto carbon-coated copper grids. The statistical analysis of the data obtained from TEM images was performed using Origin Pro software.

\subsection{3. $\mathrm{N}_{2}$ adsorption-desorption}

$\mathrm{N}_{2}$ adsorption-desorption measurements were conducted in a TriStar II Plus surface area and porosity analyzer from Micromeritics. The specific surface area of the material was determined from the adsorption-desorption isotherm by applying the BET (Brunauer-Emmett-Teller) model. The pore volume and average pore size was estimated by using the BJH (Barrett-Joyner-Halenda) model.

2.3. Doping magnetic core mesoporous silica nanoparticles with trastuzumab 
Magnetic core mesoporous silica nanoparticles were loaded with trastuzumab. In order to get the loaded magnetic core mesoporous silica nanoparticles, $200 \mu \mathrm{g}(200 \mu \mathrm{g} / 1 \mathrm{~mL})$ of Herceptin® (trastuzumab) was stirred (24 hrs) at room temperature with $100 \mu \mathrm{g}$ of magnetic core mesoporous silica nanoparticles. After this period the magnetic core mesoporous silica nanoparticles soaked with trastuzumab solution was dried under low pressure at 30 şC temperature for 12 hours, until completely dry. The dried powder containing solely magnetic core mesoporous silica nanoparticles loaded with trastuzumab were individualized and set aside for later labeling with Tc-99m. The calculation to determine the amount of trastuzumab necessary to complete dope the magnetic core mesoporous silica nanoparticles was done considering the size of the magnetic core mesoporous silica nanoparticles $(100 \mathrm{~nm})$ applied to the circumference volume (Cv) equation (1):

$$
\text { Equation 1: } C v=\pi r^{3}
$$

Where: Cv: circumference volume

$\pi$ : constant

R: Radius of the circumference

In this case, we have that the weight of one single magnetic core mesoporous silica nanoparticle is about: $1 \times 10^{-15} \mathrm{~g}$. Thus, in $100 \mu \mathrm{g}$ of magnetic core mesoporous silica nanoparticles we have approximately $10^{12-13}$ nanoparticles with a surface area approximately $872 \mathrm{~m}^{2} / \mathrm{g}$. This means that using an excess of Mab $(200 \mu \mathrm{g})$ that has a molar mass of $145.531,5 \mathrm{~g} \mathrm{~mol}^{-1}$ we have a ratio of 3:1 trastuzumab/magnetic core mesoporous silica nanoparticles. 


\subsection{Trastuzumab labeling process with Tc-99m}

The labeling process was done by the direct radiolabeling process as described previously by Pascual et al. [13], Cerqueira-Coutinho et al. [14] and Rosa et al. [15]. In this methodology we used $150 \mu \mathrm{g}$ of trastuzumab. Briefly, $100 \mu \mathrm{Ci}$ (approximately $300 \mu \mathrm{L}$ ) of Tc-99m was incubated with a stannous chloride $\left(\mathrm{SnCl}_{2}\right)$ solutions $(80 \mu \mathrm{g} / \mathrm{mL})$ (Sigma-Aldrich) for 20 minutes at room temperature. Then this solution was incubated with $150 \mu \mathrm{g}$ trastuzumab for another 10 minutes in order to label their structures.

\subsection{Quality control of the labeling process with Tc-99m}

In order to confirm the efficiency of the trastuzumab labeling process, paper chromatography was done using Whatman paper o 1 using $2 \mu$ l of the labeled-nanoparticle and acetone (Sigma-Aldrich) as mobile phase. The radioactivity of the strips was verified in a $\mathrm{y}$-counter (Perkin Elmer Wizard®) 2470, Shelton, CT City, State).

\subsection{Indirect entrapment efficiency calculation - Tc-99m EE\%}

To perform the entrapment efficiency of the trastuzumab into the magnetic core mesoporous silica nanoparticles we performed the Tc-99m entrapment efficiency. In this direction, we previously labeled the trastuzumab with Tc-99m and then, doped the magnetic core mesoporous silica nanoparticles as described in the section: doping magnetic core mesoporous silica nanoparticles with radiolabeled trastuzumab (Tc-99m-trastuzumab).

\subsection{Planar imaging from chromatographic system}


Planar images from the chromatographic system using Tc-99mtrastuzumab (3.7 MBq in $0.2 \mathrm{~mL}$ ) were done in triplicate. The images were integrated for 5 min centered at $140 \mathrm{KeV}$, with a Millennium Gamma Camera (GE Healthcare, Cleveland, USA), using a $15 \%$ window. The images were processed using OsiriX software.

\section{Statistical analysis}

Statistical analyses were performed using Origin Pro 8 (OriginLab, USA) software. Results are shown as means \pm standard deviation (S.D.). P-values less than 0.05 were considered significant.

\section{Results and discussion}

\subsection{Characterization of magnetic core MSNs}

The structure periodicity of the mesoporous material was confirmed by PXRD, which showed a sharp peak at the low-angle region for both as-made (S0-1) and calcined (S0-2) magnetic core MSNs (Figure 1). The slight shift of the peak to higher angles indicates shrinkage of the silica matrix due to the condensation of silanol groups during the calcination process. PXRD analysis at high angles confirmed the presence of magnetic cores within the structure (see inset in Figure 1).

The mesoporous structure of $\mathbf{S 0 - 2}$ magnetic core MSNs was also analysed by transmission electron microscopy and the size of the primary nanoparticles was determined by image analysis $(58.9 \pm 8.1 \mathrm{~nm}, n=100)$. The data was 
represented in a histogram, which shows the particle size distribution of the S02 nanoparticles (Figure $2 \mathrm{~A}$ and $\mathrm{B}$ ).

The $\mathrm{N}_{2}$ adsorption-desorption isotherms of the magnetic core MSNs presented a typical type IV behaviour (Figure 3), characteristic of mesoporous materials. From the isotherm curve, a specific surface area of $872 \mathrm{~m}^{2} / \mathrm{g}$ was estimated along with a pore volume of $0.85 \mathrm{~cm}^{3} / \mathrm{g}$ and an average pore diameter of 3.15 $\mathrm{nm}$.

\subsection{Trastuzumab labeling process with Tc-99m}

The trastuzumab were successfully labelled (>97\%) with Tc-99m generating a clear suspension.

\subsection{Quality control of the labeling process with Tc-99m}

The effectiveness of the labeling process was confirmed by paper chromatography which indicated almost no significant dissociation of technetium-99m from the trastuzumab for a period as long as 8 hours, as shown in Table 1:

\subsection{Planar imaging from chromatographic system}


In order to corroborate the paper chromatography a planar imaging from the paper chromatographic system has been performed, as shown in Figure 4.

The results showed an ascending movement with a great amount in the base corroborating the formation of the complex between the Tc-99m and the trastuzumab.

\subsection{Entrapment efficiency - Tc-99m EE\%}

In order to calculate the amount of Tc-99m that has been absorbed/doped into the magnetic core mesoporous silica nanoparticles (together with the trastuzumab) we calculated the entrapment efficiency (EE\%) of Tc-99m-trastuzumab into the magnetic core mesoporous silica nanoparticles, using the equation(2)::

\section{Equation 2:}

$9 \mathbf{9} m T c E E \%=\frac{\text { total amount of } T c-99 m 99 m T c \text { used }}{\text { total amount of } T c-99 m 99 m T c \text { in the supernatant }} X 10 \mathrm{o}$

The result found for the Tc-99m EE\% was $97,5 \% \pm 0,8$. This result confirmed that almost all the Tc-99m used (labelling the trastuzumab) was trapped in the surface of the mesoporous silica.

The quality control of the solely trastuzumab labeling process with Tc-99m showed that over $97 \%$ of the Tc-99m remains connected with trastuzumab for a 
period as long as 8 hours ( as shown in Table 2), corroborating our decision to use this indirect way to calculate the amount of trastuzumab.

\section{Conclusions}

The results confirmed the potential use from the indirect entrapment efficiency methodology using the radiolabeling process. This methodology although involves the use of radioactive material can be an easy, rapid and efficient methodology that can be used to calculate Mab adsorption/entrapment efficiency into nanoparticles systems.

\section{Conflict of Interest}

The authors state that do not have any conflicts of interest

\section{Acknowledgements}

The authors would like to thank the National Scientific and Technological Research Council (CNPQ) and the Rio de Janeiro State Research Foundation (FAPERJ) for funding. Authors also gratefully acknowledge the financial support from the Ministerio de Economía y Competitividad (Project MAT2012-38429C04-01) and the Generalitat Valenciana (project PROMETEO/2009/016) for support.

\section{References}


[1]. S. Santamaria, M. Delgado, L. Kremer, J.A. Garcia-Sanz, Will a mAb-Based Immunotherapy Directed against Cancer Stem Cells Be Feasible? Front Immunol., 9 ( 2017) 1509.

[2]. A.H. Staudacher, M.P. Brown, Antibody drug conjugates and bystander killing: is antigen-dependent internalisation required? Br. J. Cancer., 12 (2017) 1736-1742.

[3]. J.T. Pento, Monoclonal Antibodies for the Treatment of Cancer. Anticancer Res., 11 (2017) 5935-5939.

[4]. C. Peters, S. Brown. Antibody-drug conjugates as novel anti-cancer chemotherapeutics. Biosci. Rep., 35 (2015) e00225.

[5]. N. Diamantis, U. Banerji, Antibody-drug conjugates-an emerging class of cancer treatment. Br. J. Cancer., 114 (2016) 362-367.

[6]. H. Donaghy. Effects of antibody, drug and linker on the preclinical and clinical toxicities of antibody-drug conjugates. MAbs., 8 (2016) 659-671.

[7]. R.G. Rayavarapu, W. Petersen, C. Ungureanu, C.N. Post, T.G. van Leeuwen, S. Manohar. Synthesis and Bioconjugation of Gold Nanoparticles as Potential Molecular Probes for Light-Based Imaging Techniques. Int. J. Biomed. Imaging, (2007) 29817.

[8]. J.S. Bee, D. Chiu, S. Sawicki, J.L. Stevenson, K. Chatterjee, K. Freund, J.F. Carpenter, T.W. Randolph. Monoclonal Antibody Interactions with Micro- and Nanoparticles: Adsorption, Aggregation and Accelerated Stress Studies. J. Pharm. Sci., 98 ( 2009) 3218-3238. 
[9]. F. Sousa, P. Fonte, A. Cruz, P.J. Kennedy, I.M. Pinto, B. Sarmento. Polyester-Based Nanoparticles for the Encapsulation of Monoclonal Antibodies. Methods Mol. Biol.,1674 (2018) 239-253.

[10]. H.F. Liu, J. Ma, C. Winter, R. Bayer. Recovery and purification process development for monoclonal antibody production. MAbs., 2 (2010) 480-499.

[11]. F.C. Carney, E. Dremina, J.L. Snyder, Z. Lakos. The Role of Liquid Chromatography-Mass Spectrometry in the Characterization of Therapeutic Monoclonal Antibodies. Spectroscopy, 14 (2016) 42-45.

[12]. P.M. Ladwig, D.R. Barnidge, M.A.V. Willrich. Mass Spectrometry Approaches for Identification and Quantitation of Therapeutic Monoclonal Antibodies in the Clinical Laboratory. Clin. Vaccine Immunol., 24 (2017) e00545-16.

[13]. C. Cerqueira-Coutinho, L.P. Vidal, S.R. Pinto, R. Santos-Oliveira. Drug metabolism: Comparison of biodistribution profile of holmium in three different compositions in healthy Wistar rats. Applied Radiation and Isotopes. 112 (2016) 27-30.

[14]. L. Pascual, F. Sancenón, R. Martínez-Máñez, T.C. Barja-Fidalgo, S.V. da Silva, A.J. Sousa-Batista, C. Cerqueira-Coutinho, R. Santos-Oliveira. Mesoporous silica as multiple nanoparticles systems for inflammation imaging as nano-radiopharmaceuticals. Microporous and Mesoporous Materials. 239 (2017) 426-431.

[15]. T.G. Rosa, S.N. Dos Santos, T. de Jesus Andreoli Pinto, D.D.M. Ghisleni, T.C. Barja-Fidalgo, E. Ricci-Junior, M. Al-Qahtani, J. Kozempel, E.S. Bernardes, R. Santos-Oliveira. Microradiopharmaceutical for Metastatic Melanoma. Pharm. Res. 34 (2017) 2922-2930.

\section{Figures}




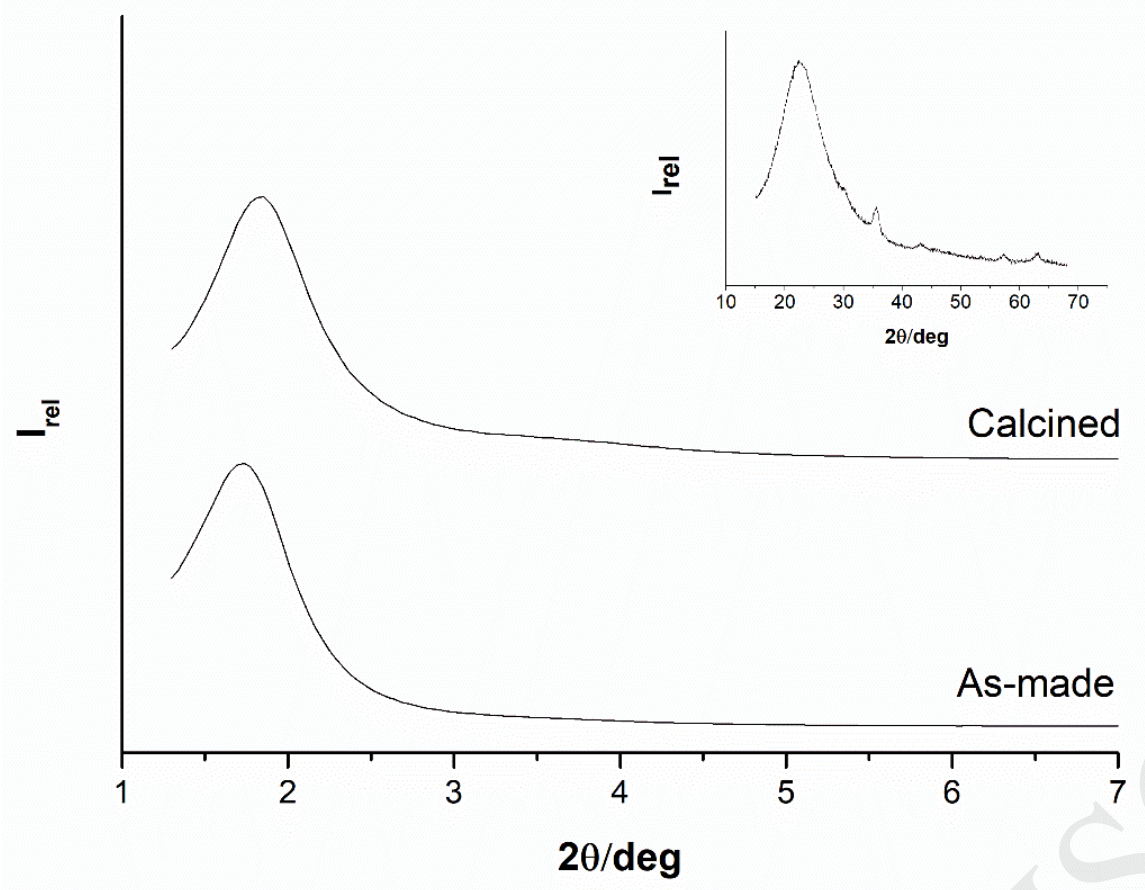

Figure 1. Powder X-ray diffraction patterns of (bottom) as-made magnetic core MSNs (S0-1) and calcined magnetic core MSNs (S0-2). Inset shows the peaks corresponding to magnetite nanocrystals and the characteristic broad peak of amorphous silica.

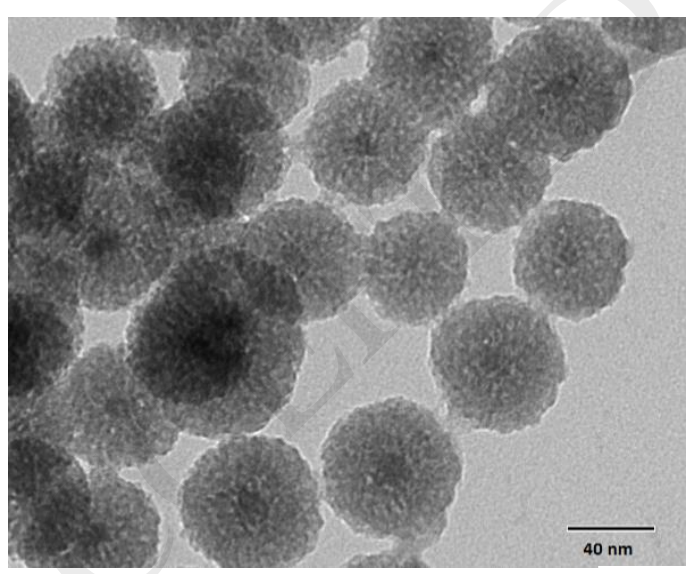

A

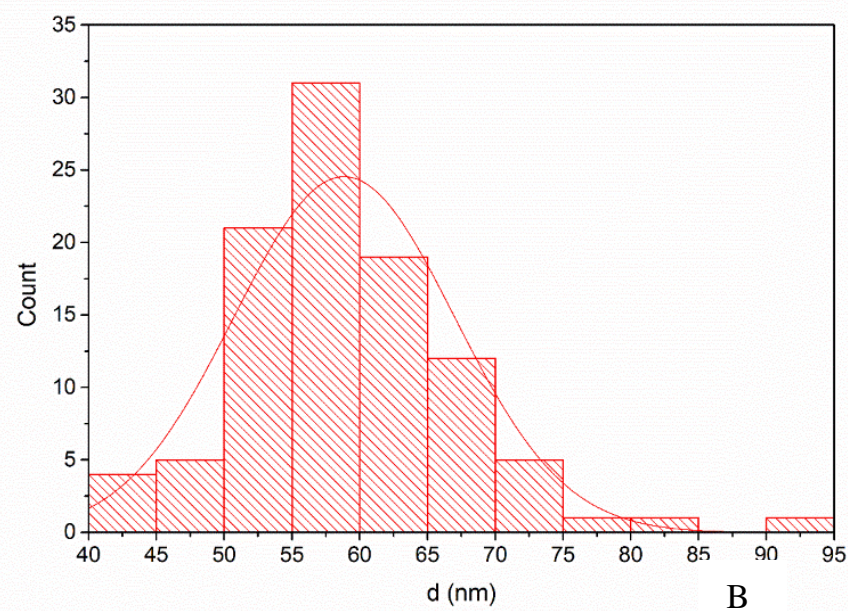

B

Figure 2. A: Transmission electron microscopy images of calcined $\mathbf{5 0 - 2}$ nanoparticles, showing the spherical shape and the magnetic core. B: Histogram and normal size distribution of calcined $\mathbf{S 0 - 2}$ nanoparticles determined by transmission electron microscopy image analysis $(n=100)$. 


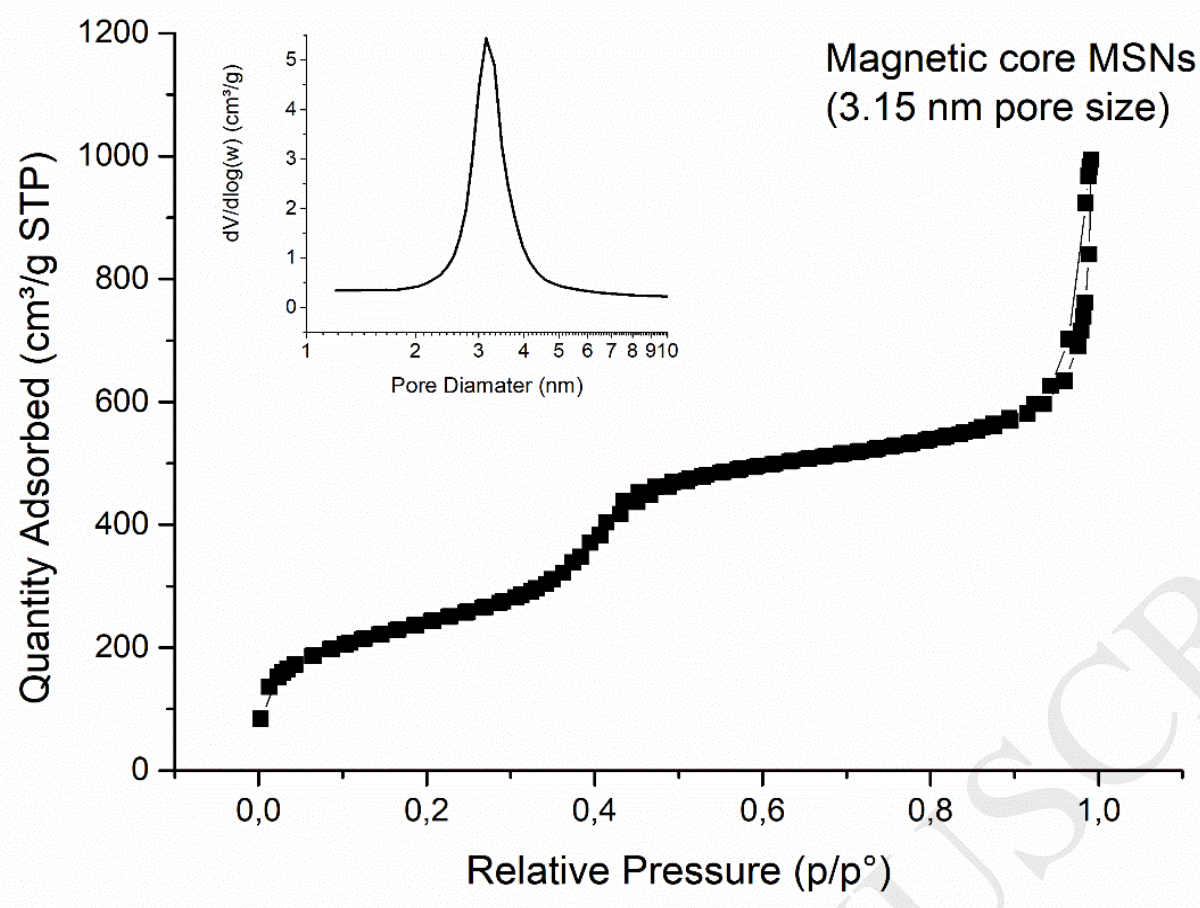

Figure 3. $\mathrm{N}_{2}$ adsorption-desorption isotherm of calcined S0-2 nanoparticles. Inset shows the pore size distribution of the material.

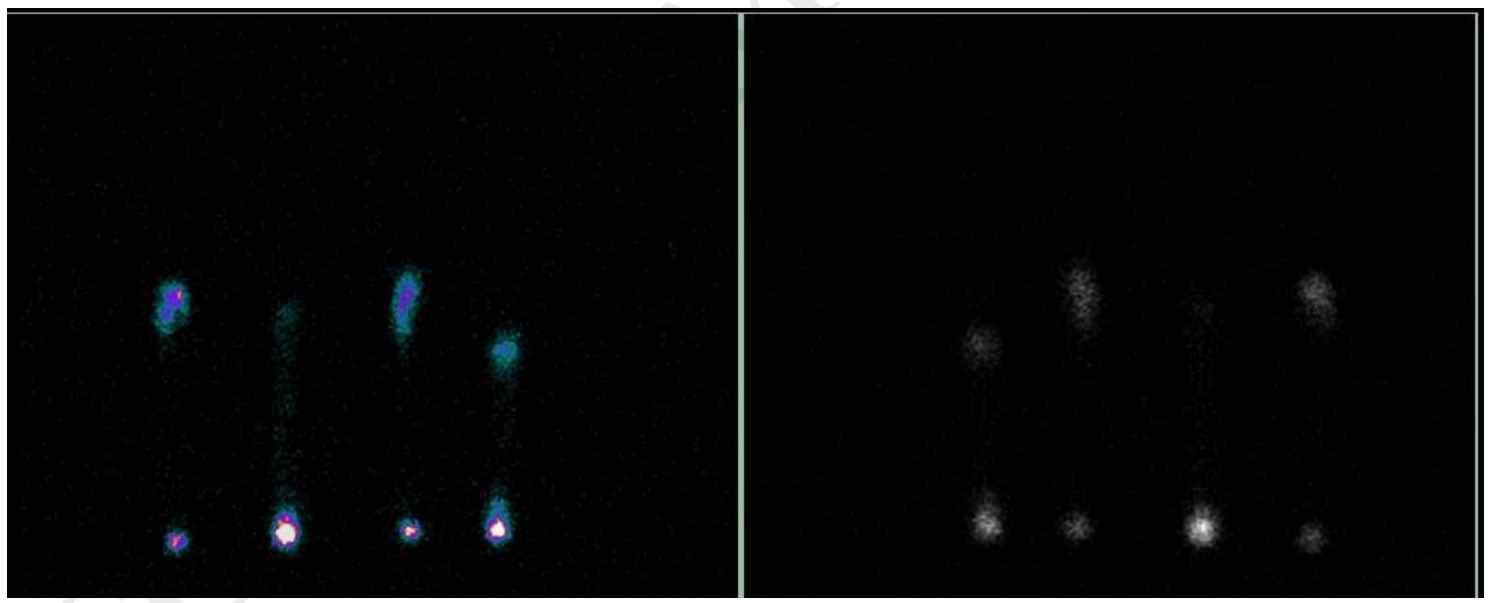

Figure 4. Planar imaging from the chromatographic system corroborating the formation of the complex Tc-99m-trastuzumab and the quality control from the labeling process. 
Table 1. Percentage of labeled trastuzumab observed over time, after ascending chromatograms of Tc-99m compared with free pertechnetate (NaTc$\left.99 \mathrm{mO}_{4}^{-}\right)$.

\begin{tabular}{|c|c|}
\hline Time (h) & Labeling (\%)Trastuzumab \\
\hline 0 & $98.6 \pm 0.7 \%$ \\
\hline 1 & $97.8 \pm 0.5 \%$ \\
\hline 2 & $97.3 \pm 1.0 \%$ \\
\hline 4 & $97.0 \pm 0.8 \%$ \\
\hline 8 & $97.1 \pm 0.5 \%$ \\
\hline
\end{tabular}

Table 2: Percentage of labeled trastuzumab observed over time, after ascending chromatograms of Tc-99m compared with free pertechnetate (NaTc$\left.99 \mathrm{mO}_{4}^{-}\right)$.

\begin{tabular}{|l|l|}
\hline Time (h) & $\begin{array}{l}\text { Labeling (\%) } \\
\text { Trastuzumab }\end{array}$ \\
\hline 0 & $99.1 \pm 0.4 \%$ \\
\hline 1 & $98.7 \pm 0.9 \%$ \\
\hline 2 & $97.9 \pm 0.8 \%$ \\
\hline 4 & $98.4 \pm 1.1 \%$ \\
\hline 8 & $97.5 \pm 0.5 \%$ \\
\hline
\end{tabular}

